\title{
Editorial
}

\section{THE COLLOQUIUM ON MULTI-SOURCE IMPERFECT INFORMATION FUSION AND ITS APPLICATION}

DOI $10.1007 / \mathrm{s} 11767-009-1001-7$

Attributes and characteristics of objects and environments can be described qualitatively and quantitatively by multi-source information. Because of the complexity of environments, limitation of sensor performance and imperfection of information acquisition technique, multi-source information is usually uncertain, vague and incomplete. The multi-source information can be described and modeled by some theories, such as fuzzy set theory, rough set theory, Bayes reasoning, Dempster-Shafer evidence theory, possibility theory and conditional event algebra, etc. Based on these theories, multi-source information fusion has recently become a research focus. The relative fusion methodologies and technologies are widely applied in many fields including target tracking, recognition and classification, fault diagnosis, information security, reliability engineering, etc.

The theme of our colloquium is "Multi-source imperfect information fusion and its application", reflecting the fast growing interest in research, development and applications in the exciting areas of information fusion. The papers in the colloquium are the latest research results of information fusion relating to random set theory, evidence theory, fuzzy set theory, Bayes theory and integrations of these theories. The concrete contents are as follows.

In multitarget tracking, the traditional approaches focus on the association between targets and measurements. The Probability Hypotheses Density (PHD) filter based on random set theory can avoid this complicated association step to some extent. However, it is just an estimation of state point and can not form tracks. Therefore, a joint estimation algorithm of the multitarget states and tracks for the PHD filter, which can completely avoid the association step, is proposed in the paper by Liu Weifeng, et al.

Due to its flexibility to deal with uncertain information processing, Dempster-Shafer theory of evidence has been widely used in many data fusion application systems. However, how to determine Basic probability Assignment (BPA), which is the main and the first step in evidence theory, is still an open issue. To resolve the problem, Deng Yong, et al. propose a new method to obtain BPA based on the similarity measure between generalized fuzzy numbers.

As we know, when all pieces of evidence are from the same event, they may be combined better than the combination among the pieces of evidence which come from multiple events. Thus, the latter evidences should be analyzed independently. Ye Qing, et al. present a novel approach for evidence clustering using a general distance of evidence and the approach for establishing the initialization of clustering is also discussed on the basis of an improved optimal distance.

The structure of modern equipment becomes more and more complex, simultaneous faults also increase unavoidably. In order to solve simultaneous faults diagnosis, a new approach based on random sets and Dezert-Smrandache theory is proposed in the paper by Li Zhiliang, et al. The diagnosis results of motor rotor show that the proposed approach is feasible and efficient.

In object identification, natural-language information is often mathematically expressed by fuzzy membership function, which can be transformed into fuzzy evidence in Dempster-Shafer theory by $\alpha$-cut sets of random set. However, $\alpha$-cut sets have consonant constructions which are very inflexible and unreasonable in practical applications. As thus, $\mathrm{Xu}$ Xiaobin, et al. propose a method for constructing more general non-consonant focal elements based on the random set theory. Some examples are also given to show the generality and the efficiency of the new method. 